$\Rightarrow$ RADIOLABELLING

\title{
Ease-C exchange
}

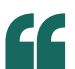

To obtain

agency approval, a

${ }^{14} \mathrm{C}$-labelled version of a drug will need to be prepared during drug development
The preparation of radiolabelled pharmaceuticals is an essential component of drug metabolism studies. Writing in the Journal of the American Chemical Society, Donald Gauthier and co-workers describe a method for facile exchange of carbon isotopes in acid chlorides.

Direct hydrogen-tritium exchange can often be achieved on the drug molecule of interest, but the exchange sites are frequently the same as those where metabolism occurs and so the labelled products are not useful in these studies. "To obtain agency approval, ${ }^{14} \mathrm{C}$-labelled version of a drug will need to be prepared during drug development," explains Gauthier. "However, this often requires the design of a bespoke synthetic route, which may differ substantially from established routes due to the limited availability of labelled starting materials."

The goal then was a method to introduce labelled carbon into a drug molecule as late as possible in the synthesis - minimizing both the time and the need to handle radioactive materials. "Carboxylic acids and their derivatives are widely found in drug molecules, so we felt that reversible insertion of $\mathrm{CO}$ into metal carbon bonds

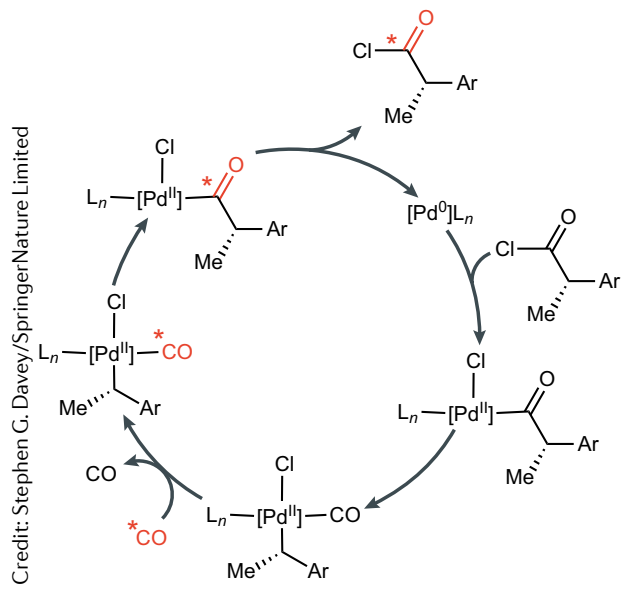

seemed to offer an opportunity to address the problem," says Gauthier. "Direct carbonyl exchange has been explored previously on simple carboxylic acids, but this required harsh (high-temperature) reaction conditions and a vast excess of precious labelled reagent."

The proposed reaction would follow a relatively simple mechanism: oxidative addition of an activated carboxylic acid derivative (such as an acid chloride) to a $\mathrm{Pd}(\mathrm{o})$ catalyst would result in formation of a Pd(II) acyl complex. Decarbonylation would give a Pd(II) alkyl complex, and the coordinated $\mathrm{CO}$ could be easily exchanged with labelled $\mathrm{CO}$ in the reaction. Reinsertion of the $\mathrm{CO}$ into the Pd-alkyl followed by reductive elimination would regenerate the acid chloride, but potentially with a labelled carbon installed.

The team was confident that an oxidative addition presented few problems, but at the outset was less certain of the reverse reaction - the reductive elimination. An initial screen for suitable reaction conditions used dihydrocinnamoyl chloride as the substrate and ${ }^{13} \mathrm{CO}$ gas (in place of the precious ${ }^{14} \mathrm{CO}$ ). A variety of phosphine ligands were examined in combination with tris (dibenzylideneacetone)dipalladium(0) $\left(\mathrm{Pd}_{2} \mathrm{dba}_{3}\right)$ as a palladium precursor, with tri-ortho-tolylphosphine providing the best result.

With this initial proof of concept in hand, Gauthier and co-workers swapped the use of CO gas for labelled COgen (a commercially available labelled acid chloride) that can be used to generate in situ a known small quantity of labelled CO. A twochamber reactor is used, with labelled $\mathrm{CO}$ gas being generated in one chamber and reacted in the second. "From a practical standpoint, this is crucial to the operational simplicity of the method as it eliminates the burden of transferring small amounts of ${ }^{14} \mathrm{CO}$ gas," he says. "We are able to transfer precisely 1.5 equivalents of the precious labelled material. If the exchange reaction reaches equilibrium then we can achieve up to $60 \%$ enrichment in labelled product but this is more than suitable for most metabolism experiments."

Tri-ortho-tolylphosphine proved a good ligand for the reaction of aromatic and primary aliphatic acid chlorides and was further applied to a number of real drug substrates or advanced drug precursors including, among others, the immediate precursor of atorvastatin (Lipitor). However, the method performed less well with secondary, and failed with tertiary, acid chlorides.

Either the decarbonylation or the final reductive elimination steps were suspected of being ratedetermining and so Gauthier and co-workers examined the use of more bulky electron-rich phosphines. The use of bulky biaryl phosphine JohnPhos as ligand or preformed bis (tri-tert-butylphosphino) palladium(0) resulted in successful exchange reactions on secondary and even tertiary acid chlorides. "We were pleased to find that the stereochemical integrity was maintained even in chiral benzylic acids such as ibuprofen, which was initially problematic," says Gauthier.

In the future, the team hope to explore methods to perform carbon isotope exchange using ${ }^{14} \mathrm{CO}_{2}-$ the universal carbon-14 starting material. Indeed, this is a promising avenue for research: during preparation of this highlight, a preprint article appeared describing a nickel-catalysed decarboxylative carboxylation.

Stephen G. Davey

ORIGINAL ARTICLE Gauthier, D. R. Jr et al. Palladium-catalyzed carbon isotope exchange on aliphatic and benzoic acid chlorides. J.Am. Chem. Soc. https://doi.org/10.1021/jacs.8b09808 (2018) FURTHER READING Kingston, C. et al. Direct carbon isotope exchange through decarboxylative carboxylation. https://doi.org/10.26434/chemrxiv. 7318871.v1 (2018) 